\title{
PHYSICAL DISSIPATION AND THE METHOD OF CONTROLLED LAGRANGIANS
}

\author{
C. A. Woolsey ${ }^{1}$, A. M. Bloch ${ }^{2}$, \\ 1 Aerospace \& Ocean Engineering \\ Virginia Tech \\ Blacksburg, VA 24061, USA \\ fax: (540) 231-9632 \\ e-mail: cwoolsey@vt.edu \\ ${ }^{3}$ Mechanical \& Aerospace Engineering \\ Princeton University \\ Princeton, NJ 08544, USA \\ e-mail: naomieprinceton.edu
}

\author{
N. E. Leonard ${ }^{3}$ ，J. E. Marsden ${ }^{4}$ \\ 2 Dept. of Mathematics \\ University of Michigan \\ Ann Arbor, MI 48109, USA \\ e-mail: abloch@math.lsa.umich.edu \\ ${ }^{4}$ Control \& Dynamical Systems \\ California Institute of Technology 107-81 \\ Pasadena, CA 91125, USA \\ e-mail: marsdenecds.caltech.edu
}

Keywords: Stabilization of Non-linear Systems, Dissipative and Energy-based Design.

\begin{abstract}
We describe the effect of physical dissipation on stability of equilibria which have been stabilized, in the absence of damping, using the method of controlled Lagrangians. This method applies to a class of underactuated mechanical systems including "balance" systems such as the pendulum on a cart. Since the method involves modifying a system's kinetic energy metric through feedback, the effect of dissipation is obscured. In particular, it is not generally true that damping makes a feedback-stabilized equilibrium asymptotically stable. Damping in the unactuated directions does tend to enhance stability, however damping in the controlled directions must be "reversed" through feedback. In this paper, we suggest a choice of feedback dissipation to locally exponentially stabilize a class of controlled Lagrangian systems.
\end{abstract}

\section{Introduction}

The method of controlled Lagrangians is a technique for stabilizing underactuated Lagrangian mechanical systems with symmetry [4]. The method provides a feedback control law which preserves the Lagrangian structure but which shapes the energy of the closed-loop system. Standard stability analysis techniques may then be used to find conditions on control gains for closed-loop stability. Energy methods are appealing because they provide Lyapunov functions which are useful for estimating regions of attraction. Furthermore, for conservative system models, energy methods give conclusive stability results where spectral techniques fail. When physical dissipation is included, however, spectral analysis yields conditions for local exponential stability. Exponential stability is very desirable because of the inherent robustness to model uncertainty.

In this paper, we describe the effect of physical dissipation on a system which, in the conservative approximation, has been stabilized using the method of controlled Lagrangians. For the closed-loop system (under the control law implied for the conservative model), damping in the unactuated directions tends to enhance stability while damping in the actuated directions tends to destabilize. We therefore suggest a choice of feedback dissipation to exponentially stabilize the equilibrium.

We consider only equilibria that are fixed points for the unreduced equations, such as an inverted pendulum on a cart at rest. The analysis can be generalized to include relative equilibria, as well. In future work, we will also extend our treatment to include relative equilibria for systems with full configuration symmetry such as a spacecraft with internal rotors.

The idea of using feedback to shape a mechanical system's energy is well-established. Stabilization by potential energy shaping is described in [7]. Because this approach does not generally apply to underactuated systems, the idea of kinetic energy shaping has also been considered. Stabilization by kinetic shaping has been studied, in the Lagrangian setting, by Bloch et al and also in [1] and [5]. On the Hamiltonian side, the authors of [6] extend their previous work on passivity-based control techniques to include physical and feedback damping. Here, we make a similar extension to consider the effect of damping on a controlled Lagrangian system. This work relates to previous results concerning stabilization of an underwater vehicle subject to viscous forces and torques [8].

The paper is organized as follows. In Section 2 we briefly review the method of controlled Lagrangians for a class of conservative mechanical systems. In Section 3, we include physical dissipation and describe the stability result. Section 4 describes an experimental application which is a variant of the classic pendulum on a cart. We conclude in Section 5.

\section{Summary of the Method}

Consider a Lagrangian mechanical system defined on configuration space $Q$. Suppose that $Q$ is subject to the free and proper action of an abelian Lie group $G$ and that the Lagrangian $L$ is 
invariant under this action. Suppose also that control forces enter only in this symmetry $(G)$ direction. The method of controlled Lagrangians provides a choice of feedback such that the closed-loop system derives from a new Lagrangian with a control-parameterized kinetic energy. The method also allows for potential shaping although, in this paper, we consider only kinetic shaping. (See [2] for details on potential shaping for controlled Lagrangian systems.)

To understand the approach, recall that the kinetic energy metric $g(\cdot, \cdot)$ provides a natural splitting of any tangent vector $v_{q}$ into "horizontal" and "vertical" components. The vertical component of $v_{q}$ is tangent to the $G$-orbit through $q$ while the horizontal component is its metric orthogonal. That is,

$$
v_{q}=\operatorname{Hor} v_{q}+\operatorname{Ver} v_{q}
$$

for each $v_{q} \in T_{q} Q$ where

$$
g\left(v_{q}, w_{q}\right)=g\left(\text { Hor } v_{q}, \text { Hor } w_{q}\right)+g\left(\operatorname{Ver} v_{q}, \operatorname{Ver} w_{q}\right)
$$

for $v_{q}, w_{q} \in T_{q} Q$. The tangent space at each point thus decomposes into a vertical space Ver and a horizontal space Hor.

The method of controlled Lagrangians involves choosing a new horizontal space, denoted $\mathrm{Hor}_{\tau}$, a new metric $g_{\sigma}$ on horizontal vectors, and a new metric $g_{\rho}$ on vertical vectors. The parameters $\tau, \sigma$, and $\rho$ are then chosen in such a way that, under an appropriate feedback control law, the closed-loop equations are Lagrangian with respect to the modified kinetic energy metric.

Let $\mathfrak{g}$ denote the Lie algebra of $G$ and define $\tau$ as a Lie algebravalued horizontal one-form on $Q$. That is, let $\tau: T_{q} Q \rightarrow \mathfrak{g}$ be such that

$$
\tau\left(v_{q}\right)=\tau\left(\text { Hor } v_{q}\right) \in \mathfrak{g} .
$$

We denote by $\tau(v)_{Q}$ the vector field on $Q$ which is the infinitesimal generator of $\tau(v)$. One may then define the " $\tau$-horizontal space" at $q \in Q$ as the space of all tangent vectors of the form $\operatorname{Hor}_{\tau} v_{q}=$ Hor $v_{q}-\tau(v)_{Q}(q)$. The infinitesimal generator of $\tau(v)$ evaluated at $q$ is a vertical vector, so the complementary space to $\operatorname{Hor}_{\tau}$ defined by $\operatorname{Ver}_{\tau} v_{q}=\operatorname{Ver} v_{q}+\tau(v)_{Q}(q)$ is equivalent to the original vertical space.

Suppose that $\tau, g_{\sigma}$, and $g_{\rho}$ are given, that $g=g_{\sigma}$ on the horizontal space, and that the horizontal and vertical spaces are orthogonal for $g_{\sigma}$. Then the controlled Lagrangian is [4]

$$
\begin{aligned}
& L_{\tau, \sigma, \rho}\left(v_{q}\right) \\
& =\frac{1}{2}\left(g_{\sigma}\left(\operatorname{Hor}_{\tau} v_{q}, \operatorname{Hor}_{\tau} v_{q}\right)+g_{\rho}\left(\operatorname{Ver}_{\tau} v_{q}, \operatorname{Ver}_{\tau} v_{q}\right)\right)-V(q) \\
& =L\left(v_{q}+\tau(v)_{Q}(q)\right)+\frac{1}{2} g_{\sigma}\left(\tau(v)_{Q}(q), \tau(v)_{Q}(q)\right) \\
& \quad+\frac{1}{2}\left(g_{\rho}-g\right)\left(\operatorname{Ver}_{\tau} v_{q}, \operatorname{Ver}_{\tau} v_{q}\right) .
\end{aligned}
$$

Under certain conditions on the parameters $\tau, \sigma$, and $\rho$ and the metric $g$, there is a control law for which the closed-loop equations under the prescribed control law are simply Lagrange's equations for $L_{\tau, \sigma, \rho}$. These conditions typically leave some freedom in the parameters $\tau, \sigma$, and $\rho$, which then play the role of control gains.

The system state, which corresponds to a point in $Q$, can be described by an element in $G$ and an element in the complementary shape space $Q / G$. Define local coordinates $\theta^{a}$ for $G$ and $x^{\alpha}$ for $Q / G$. In these coordinates, the Lagrangian is $L\left(x^{\alpha}, \dot{x}^{\alpha}, \dot{\theta}^{a}\right)=$

$$
\frac{1}{2} g_{\alpha \beta} \dot{x}^{\alpha} \dot{x}^{\beta}+g_{\alpha a} \dot{x}^{\alpha} \dot{\theta}^{a}+\frac{1}{2} g_{a b} \dot{\theta}^{a} \dot{\theta}^{b}-V\left(x^{\alpha}\right)
$$

where $g_{\alpha \beta}, g_{\alpha b}$, and $g_{a b}$ are the local components of the kinetic energy metric $g(\cdot, \cdot)$. The Euler-Lagrange equations are

$$
\begin{aligned}
\frac{d}{d t} \frac{\partial L}{\partial \dot{x}^{\alpha}}-\frac{\partial L}{\partial x^{\alpha}} & =0 \\
\frac{d}{d t} \frac{\partial L}{\partial \dot{\theta}^{a}} & =u_{a} .
\end{aligned}
$$

The method of controlled Lagrangians provides a technique for stabilizing an unstable equilibrium

$$
\left(x^{\alpha}, \dot{x}^{\alpha}, \dot{\theta}^{a}\right)_{e}=\left(x_{e}^{\alpha}, 0,0\right)
$$

for equations (2). If (2) describes a balance system, $x_{e}^{\alpha}$ is a local maximum of the potential $V\left(x^{\alpha}\right)$. Because of limited control authority, one cannot stabilize by shaping the potential energy to make the equilibrium a minimum. However, one may shape the kinetic energy so that the equilibrium becomes a maximum of the modified total energy. In the conservative setting, at least, this is sufficient for stability.

Let $\tau_{\alpha}^{b}$ be the local representation of $\tau$ and let $\sigma_{a b}$ and $\rho_{a b}$ denote the $a b$ components of $g_{\sigma}$ and $g_{\rho}$, respectively. Four conditions which are sufficient for matching are [3]

Assumption GM-1. $\tau_{\alpha}^{b}=-\sigma^{a b} g_{b \alpha}$.

Assumption GM-2. $\sigma^{b d}\left(\sigma_{a d, \alpha}+g_{a d, \alpha}\right)=2 g^{b d} g_{a d, \alpha}$.

Assumption GM-3. $\left(\rho_{a b}-g_{a b}\right)_{, \alpha}=0$.

Assumption GM-4. Letting $\zeta_{\alpha}^{a}=g^{a c} g_{c \alpha}$,

$$
\begin{aligned}
0= & \tau_{\alpha, \delta}^{b}-\tau_{\delta, \alpha}^{b}+\rho^{b a} \varpi_{a c}\left(\zeta_{\alpha, \delta}^{c}-\zeta_{\delta, \alpha}^{c}\right) \\
& -\rho^{b a} g_{a c, \delta} \rho^{c d} \varpi_{d a} \zeta_{\alpha}^{a}-\rho^{b a} g_{a c, \alpha} \tau_{\delta}^{a} .
\end{aligned}
$$

Commas in the subscripts denote partial derivatives.

Conditions GM-1 through GM-4 are referred to as the "general matching conditions" to distinguish them from certain special cases. These conditions impose requirements on the control parameters $\tau, \sigma$, and $\rho$ and on the unmodified kinetic energy metric $g$.

Using Assumption GM-1, the coordinate expression for the controlled Lagrangian (1) becomes $L_{\tau, \sigma, \rho}\left(x^{\alpha}, \dot{x}^{\alpha}, \dot{\theta}^{a}\right)=$

$\frac{1}{2}\left(g_{\tau, \sigma, \rho}\right)_{\alpha \beta} \dot{x}^{\alpha} \dot{x}^{\beta}+\left(g_{\tau, \sigma, \rho}\right)_{\alpha b} \dot{x}^{\alpha} \dot{\theta}^{b}+\frac{1}{2}\left(g_{\tau, \sigma, \rho}\right)_{a b} \dot{\theta}^{a} \dot{\theta}^{b}-V\left(x^{\alpha}\right)$

where

$$
\left(g_{\tau, \sigma, \rho}\right)_{\alpha \beta}=\left(\begin{array}{c}
g_{\alpha \beta}-g_{\alpha c}\left(g^{c d}-\sigma^{c d}\right) g_{d \beta}+ \\
g_{\alpha c}\left(g^{c d}-\sigma^{c d}\right) \rho_{d e}\left(g^{e f}-\sigma^{e f}\right) g_{f \beta}
\end{array}\right)
$$




$$
\begin{aligned}
\left(g_{\tau, \sigma, \rho}\right)_{\alpha b} & =g_{\alpha c}\left(g^{c d}-\sigma^{c d}\right) \rho_{d b} \\
\left(g_{\tau, \sigma, \rho}\right)_{a b} & =\rho_{a b} .
\end{aligned}
$$

Under the matching conditions, the control law prescribed by the method of controlled Lagrangians gives the closed-loop equations

$$
\begin{aligned}
\frac{d}{d t} \frac{\partial L_{\tau, \sigma, \rho}}{\partial \dot{x}^{\alpha}}-\frac{\partial L_{\tau, \sigma, \rho}}{\partial x^{\alpha}} & =0 \\
\frac{d}{d t} \frac{\partial L_{\tau, \sigma, \rho}}{\partial \dot{\theta}^{a}} & =0 .
\end{aligned}
$$

Comparing the open-loop system (2) and the closed-loop system (4), one may verify that the control law is $u_{a}=$

$$
-\frac{d}{d t}\left\{\left(\rho_{a b}-g_{a b}\right) \dot{\theta}^{b}+\left(\rho_{a c}\left(g^{c d}-\sigma^{c d}\right) g_{d \beta}-g_{a \beta}\right) \dot{x}^{\beta}\right\} .
$$

Remark 2.1 The control law (5) involves acceleration as well as position and velocity. One may use the equations of motion to eliminate acceleration from the control law. When dissipation is included in the model, the control law (5) includes contributions from the dissipative forces. However, the control law obtained by using the conservative equations of motion to eliminate acceleration does not involve these external forces. We assume throughout that this latter control law is applied so that $u_{a}$ does not depend on physical or feedback dissipation.

Note from the closed-loop equations (4) that the controlled momentum conjugate to $\theta^{a}$ is conserved. The conservation law will no longer hold when we include physical and feedback dissipation, however it is useful for constructing a Lyapunov function for the conservative system. Define

$$
\tilde{J}_{a}=\frac{\partial L_{\tau, \sigma, \rho}}{\partial \dot{\theta}^{a}}=\rho_{a b}\left(\dot{\theta}^{b}+\left(g^{b c}-\sigma^{b c}\right) g_{c \alpha} \dot{x}^{\alpha}\right) .
$$

Under the control law (5), the desired equilibrium (3) will be stable provided the control-modified energy

$$
E_{\tau, \sigma, \rho}\left(x^{\alpha}, \dot{x}^{\alpha}\right)=\frac{1}{2} A_{\alpha \beta} \dot{x}^{\alpha} \dot{x}^{\beta}+\frac{1}{2} \rho^{a b} \tilde{J}_{a} \tilde{J}_{b}+V\left(x^{\alpha}\right)
$$

is definite. The tensor $A_{\alpha \beta}$ is the coordinate form of the modified horizontal kinetic energy metric $g_{\sigma}$,

$$
A_{\alpha \beta}=g_{\alpha \beta}-g_{\alpha a}\left(g^{a b}-\sigma^{a b}\right) g_{b \beta} .
$$

The energy $E_{\tau, \sigma, \rho}$ is simply the Routhian of $L_{\tau, \sigma, \rho}$.

In analyzing stability of the conservative system, one may treat $E_{\tau, \sigma, \rho}$ as a function of $x^{\alpha}$ and $\dot{x}^{\alpha}$, parameterized by $\tilde{J}_{a}$. The modified energy serves as a Lyapunov function candidate on level sets of $\tilde{J}_{a}$. Assuming that $x_{e}^{\alpha}$ is a maximum of the amended potential

$$
V_{\mu}\left(x^{\alpha}\right)=\frac{1}{2} \rho^{a b} \tilde{J}_{a} \tilde{J}_{b}+V\left(x^{\alpha}\right),
$$

the equilibrium (3) is stable if $\left.A_{\alpha \beta}\right|_{e}$ is negative definite.
Alternatively, one may treat $E_{\tau, \sigma, \rho}$ as a function of $\tilde{J}_{a}$, as well, and use the energy-Casimir method (for example) to construct a Lyapunov function. Such a function can be useful for studying closed-loop stability under the influence of dissipative forces which can destroy conservation of $\tilde{J}_{a}$.

\section{Physical and Feedback Dissipation}

To determine the effect of physical and feedback dissipation on the feedback-controlled system (4), consider the following open-loop equations obtained by appending generalized forces to equations (2):

$$
\begin{aligned}
\frac{d}{d t} \frac{\partial L}{\partial \dot{x}^{\alpha}}-\frac{\partial L}{\partial x^{\alpha}} & =F_{\alpha} \\
\frac{d}{d t} \frac{\partial L}{\partial \dot{\theta}^{a}} & =u_{a}+F_{a}
\end{aligned}
$$

The generalized forces $F_{\alpha}$ and $F_{a}$ might represent physical dissipation, additional control, etc. Let square brackets denote the matrix form of a tensor and define the generalized inertia matrices

$$
\boldsymbol{M}=\left(\begin{array}{ll}
{\left[g_{\alpha \beta}\right]} & {\left[g_{\alpha b}\right]} \\
{\left[g_{a \beta}\right]} & {\left[g_{a b}\right]}
\end{array}\right)
$$

and

$$
\boldsymbol{M}_{\tau, \sigma, \rho}=\left(\begin{array}{ll}
{\left[\left(g_{\tau, \sigma, \rho}\right)_{\alpha \beta}\right]} & {\left[\left(g_{\tau, \sigma, \rho}\right)_{\alpha b}\right]} \\
{\left[\left(g_{\tau, \sigma, \rho}\right)_{a \beta}\right]} & {\left[\left(g_{\tau, \sigma, \rho}\right)_{a b}\right]}
\end{array}\right)
$$

Assuming that $u_{a}$ is chosen as described in Remark 2.1, the equations of motion become

$$
\left(\begin{array}{c}
{\left[\frac{d}{d t} \frac{\partial L_{\tau, \sigma, \rho}}{\partial \dot{x}^{\alpha}}-\frac{\partial L_{\tau, \sigma, \rho}}{\partial x^{\alpha}}\right]} \\
{\left[\frac{d}{d t} \frac{\partial L_{\tau, \sigma, \rho}}{\partial \dot{\theta}^{a}}\right]}
\end{array}\right)=M_{\tau, \sigma, \rho} M^{-1}\left(\begin{array}{c}
{\left[F_{\alpha}\right]} \\
{\left[F_{a}\right]}
\end{array}\right) .
$$

Once again, consider stability of the equilibrium (3) under the controlled Lagrangian control law with $F_{\alpha}=F_{a}=0$. Suppose that a Lyapunov function has been constructed, for this conservative setting, using the modified system energy $E_{\tau, \sigma, \rho}$ and the conserved momentum $\tilde{J}_{a}$. When damping is included, one may use this function to search for conditions for asymptotic stability. This approach succeeds when there is feedback dissipation but no physical damping $[2,4]$. Unfortunately, the approach is not so useful when there is physical dissipation; generic damping makes the Lyapunov rate indefinite in a way that feedback dissipation cannot compensate. However, local analysis yields a definitive stability result.

Changing coordinates from $\left(x^{\alpha}, \dot{x}^{\alpha}, \dot{\theta}^{a}\right)$ to $\left(x^{\alpha}, \dot{x}^{\alpha}, \tilde{J}_{a}\right)$, we consider the equilibrium

$$
\left(x^{\alpha}, \dot{x}^{\alpha}, \tilde{J}_{a}\right)_{e}=\left(x_{e}^{\alpha}, 0,0\right)
$$

corresponding to (3). Before linearizing about this equilibrium, it is convenient to define

$$
\begin{aligned}
B_{\alpha \beta} & =g_{\alpha \beta}-g_{\alpha a} g^{a b} g_{b \beta}>0 \\
D^{a b} & =g^{a b}+\sigma^{a c} g_{c \alpha} B^{\alpha \beta} g_{\beta e} g^{e b} .
\end{aligned}
$$


The linearized dynamics are

$$
\begin{aligned}
\delta \ddot{x}^{\alpha}= & -\left(A^{\alpha \gamma} \frac{\partial^{2} V}{\partial x^{\gamma} \partial x^{\beta}}\right)_{e} \delta x^{\beta}+\left(B^{\alpha \beta}\right)_{e} \delta F_{\beta} \\
& -\left(B^{\alpha \beta} g_{\beta c} g^{c b}\right)_{e} \delta F_{b} \\
\delta \tilde{J}_{a}= & \left(\rho_{a c} D^{c b}\right)_{e} \delta F_{b}-\left(\rho_{a c} \sigma^{c d} g_{d \gamma} B^{\gamma \beta}\right)_{e} \delta F_{\beta}
\end{aligned}
$$

We assume that $\delta F_{\alpha}=-d_{\alpha \beta} \delta \dot{x}^{\beta}$ where $d_{\alpha \beta}>0$ is a symmetric damping tensor. That is, we assume the linearized dissipative force in the unactuated directions is nonzero. (Certainly $F_{\alpha}$ may include terms which are higher order in velocity.)

We assume that any physical damping in the actuated directions can be exactly cancelled so that $\delta F_{a}$ may be specified as a control. Suppose that

$$
\delta F_{a}=\left(g_{a b}\right)_{e} \tilde{d}_{x}^{b c}\left(g_{c \beta}\right)_{e} \delta \dot{x}^{\beta}-\left(D_{a b} \rho^{b c} g_{c d}\right)_{e} \tilde{d}_{J}^{d e} \delta \tilde{J} .
$$

where $\tilde{d}_{x}^{a b}$ and $\tilde{d}_{J}^{a b}$ are symmetric dissipative control gain matrices. Through coupling, the term $\tilde{d}_{x}^{a b}$ enhances the damping in the unactuated directions while $\tilde{d}_{J}^{d e}$ provides damping in the controlled directions.

The complete linearized dynamics take the form

$$
\left(\begin{array}{c}
{\left[\delta \dot{x}^{\alpha}\right]} \\
{\left[\delta \ddot{x}^{\alpha}\right]} \\
{\left[\delta \tilde{\tilde{J}}_{a}\right]}
\end{array}\right)=\left(\begin{array}{ccc}
\mathbf{0} & \mathcal{I} & \mathbf{0} \\
\boldsymbol{A} & \boldsymbol{B} & \boldsymbol{C} \\
\mathbf{0} & \boldsymbol{D} & \boldsymbol{E}
\end{array}\right)\left(\begin{array}{l}
{\left[\delta x^{\alpha}\right]} \\
{\left[\delta \dot{x}^{\alpha}\right]} \\
{\left[\delta \tilde{J}_{a}\right]}
\end{array}\right)
$$

where $\mathcal{I}$ is the $3 \times 3$ identity matrix and

$$
\begin{aligned}
\boldsymbol{A} & =\left[-A^{\alpha \gamma} \frac{\partial^{2} V}{\partial x^{\gamma} \partial x^{\beta}}\right]_{e} \\
\boldsymbol{B} & =\left[-B^{\alpha \gamma}\left(d_{\gamma \beta}+g_{\gamma c} \tilde{d}_{x}^{c d} g_{d \beta}\right)\right]_{e} \\
\boldsymbol{C} & =\left[B^{\alpha \gamma} g_{\gamma c} g^{c d} D_{d e} \rho^{e f} g_{f h} \tilde{d}_{J}^{h b}\right]_{e} \\
\boldsymbol{D} & =\left[\rho_{a c}\left(\sigma^{c d} g_{d \gamma} B^{\gamma \psi} d_{\psi \beta}+D^{c d} g_{d e} \tilde{d}_{x}^{e f} g_{f \beta}\right)\right]_{e} \\
\boldsymbol{E} & =\left[-g_{a c} \tilde{d}_{J}^{c b}\right]_{e} .
\end{aligned}
$$

One might expect that $\boldsymbol{A}<0$ and that $\boldsymbol{B}<0$ whenever $\tilde{d}_{x}^{a b} \geq$ 0 . (If $\left[A^{\alpha \beta}\right]_{e}<0$ commutes with $\left[V_{, \alpha, \beta}\right]_{e}<0$, then $\boldsymbol{A}=$ $\boldsymbol{A}^{T}<0$. A similar statement applies for $\boldsymbol{B}$.) In this case, one can show that

$$
\left(\begin{array}{ll}
0 & \mathcal{I} \\
A & B
\end{array}\right)
$$

is Hurwitz.

Suppose that we choose $\tilde{d}_{J}^{a b}=0$. Then $\boldsymbol{C}=\mathbf{0}$ and $\boldsymbol{E}=\mathbf{0}$, so the linearized system is neutrally stable with $m$ zero eigenvalues, where $m=\operatorname{dim}\left(\left[\delta \tilde{J}_{a}\right]\right)$. The problem is to choose $\tilde{d}_{J}^{a b}$ to drive these $m$ eigenvalues into the open left half plane without driving the formerly stable eigenvalues into the right half plane. Inspection of $\boldsymbol{E}$ suggests choosing $\tilde{d}_{J}^{a b}>0$. If $\left[\tilde{d}_{J}^{a b}\right]>0$ commutes with $\left[g_{a b}\right]_{e}>0$ then $\boldsymbol{E}=\boldsymbol{E}^{T}<0$ and thus $\boldsymbol{E}$ is Hurwitz. Unfortunately, because of the coupling terms $\boldsymbol{C}$ and $\boldsymbol{D}$, it is not sufficient for exponential stability that $\boldsymbol{E}$ be Hurwitz. One must determine more explicit conditions on $\tilde{d}_{J}^{a b}$ to conclude exponential stability. For specific problems, techniques such as the Routh-Hurwitz method can provide such conditions.

Remark 3.1 Damping in the uncontrolled directions actually enhances stability. This follows by observing that the submatrix (13) is Hurwitz even with $\tilde{d}_{x}^{b c}=0$. On the other hand, requiring that $\tilde{d}_{J}^{a b}>0$ equates to "reversing" the damping in the controlled directions. This would imply that generic damping in these directions is detrimental to stability.

Remark 3.2 Because of symmetry, we have ignored $\theta^{a}$ in the analysis. Therefore, we do not expect $\theta^{a}$ to approach a specific value. By adding an appropriate symmetry-breaking potential control law (i.e., a fictitious spring force), one could presumably obtain local exponential stability to a particular point. (See [2].)

\section{Example: The Pendulum on a Rotor Arm}

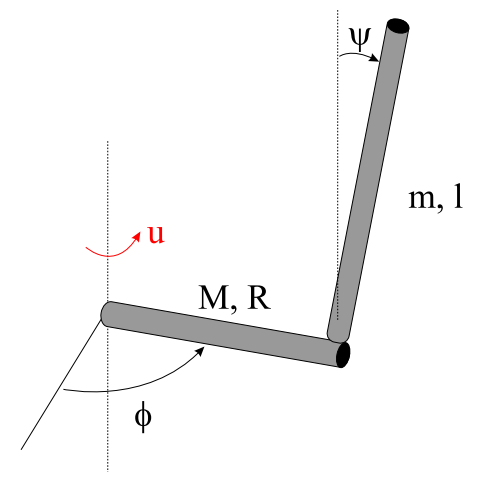

Figure 1: Pendulum on a rotary arm.

In this section, we consider the pendulum on a rotor arm. This problem was treated in [3], although the model used here differs slightly to better approximate an experimental apparatus used to test the theory. The previous model considered point masses linked by massless rods whereas the present model involves two rods with uniformly distributed mass. Figure 1 depicts the device and the choice of coordinates. The Lagrangian for the uncontrolled system is

$$
L(\psi, \dot{\psi}, \dot{\phi})=\frac{1}{2} g_{\alpha \beta} \dot{\psi}^{2}+g_{\alpha \beta} \dot{\psi} \dot{\phi}+\frac{1}{2} g_{a b} \dot{\phi}^{2}-m g l(\cos \psi-1)
$$

where, in a slight abuse of notation, we define

$$
\begin{aligned}
g_{\alpha \beta} & =\frac{1}{3} m l^{2}, \\
g_{\alpha b} & =\frac{1}{2} m l R \cos \psi, \quad \text { and } \\
g_{a b} & =\left(\frac{1}{3} M+m\right) R^{2}+\frac{1}{3} m l^{2} \sin ^{2} \psi .
\end{aligned}
$$

A control torque is applied to the horizontal link about the vertical axis. Assuming that no external forces act, other than the 
control torque $u$, the Euler-Lagrange equations are

$$
\begin{aligned}
\frac{d}{d t} \frac{\partial L}{\partial \dot{\psi}}-\frac{\partial L}{\partial \psi} & =0 \\
\frac{d}{d t} \frac{\partial L}{\partial \dot{\phi}} & =u .
\end{aligned}
$$

In [3], the matching conditions suggested choosing

$$
\sigma_{a b}=g_{a c} k^{c d} g_{d b}+g_{a b} \quad \text { and } \quad \rho_{a b}=g_{a b}+k_{a b}
$$

where $k_{a b}$ represents a constant gain. Let $k_{a b}=k$ for brevity.

In the conservative setting, the feedback control law

$$
\begin{aligned}
& u=u_{\mathrm{cL}}=-k \ddot{\phi}= \\
& -k \frac{\frac{1}{2} m l R \sin \psi \dot{\psi}^{2}-m l^{2} \sin 2 \psi\left(\frac{1}{3} \dot{\psi} \dot{\phi}+\frac{1}{4} \frac{R}{l}\left(\cos \psi \dot{\phi}^{2}+\frac{g}{l}\right)\right)}{\left(\frac{1}{3} M+\frac{1}{4} m\right) R^{2}+m\left(\frac{3}{4} R^{2}+\frac{1}{3} l^{2}\right) \sin ^{2} \psi+k}
\end{aligned}
$$

leads to the modified Euler-Lagrange equations

$$
\begin{aligned}
\frac{d}{d t} \frac{\partial L_{\tau, \sigma, \rho}}{\partial \dot{\psi}}-\frac{\partial L_{\tau, \sigma, \rho}}{\partial \psi} & =0 \\
\frac{d}{d t} \frac{\partial L_{\tau, \sigma, \rho}}{\partial \dot{\phi}} & =0
\end{aligned}
$$

with controlled Lagrangian

$$
L_{\tau, \sigma, \rho}(\psi, \dot{\psi}, \dot{\phi})=L(\psi, \dot{\psi}, \dot{\phi})+\frac{1}{2} k \dot{\phi}^{2}
$$

Define the controlled momentum conjugate to $\phi, \tilde{J}=$

$$
\left(\left(\frac{1}{3} M+m\right) R^{2}+\frac{1}{3} m l^{2} \sin ^{2} \psi+k\right) \dot{\phi}+\frac{1}{2} m l R \cos \psi \dot{\psi}
$$

The momentum $\tilde{J}$ is conserved in the absence of dissipation. The modified system energy is

$$
E_{\tau, \sigma, \rho}(\psi, \dot{\psi})=\frac{1}{2} A_{\alpha \beta} \dot{\psi}^{2}+\frac{\tilde{J}^{2}}{2 \rho_{a b}}+m g l(\cos \psi-1)
$$

where

$$
A_{\alpha \beta}=\frac{1}{3} m l^{2}-\frac{\left(\frac{1}{2} m l R \cos \psi\right)^{2}}{\left(\frac{1}{3} M+m\right) R^{2}+\frac{1}{3} m l^{2} \sin ^{2} \psi+k} .
$$

For the conservative system model, the equilibrium

$$
(\psi, \dot{\psi}, \tilde{J})_{e}=(0,0,0) .
$$

is stable provided $E_{\tau, \sigma, \rho}$ is definite. Define the amended potential

$$
V_{\mu}(\psi)=m g l(\cos \psi-1)+\frac{1}{2} \frac{\tilde{J}^{2}}{(M+m) R^{2}+m l^{2} \sin ^{2} \psi+k} .
$$

The equilibrium (16) will be stable provided

$$
\operatorname{sign}\left(A_{\alpha \beta}\right)_{e}=\operatorname{sign}\left(\frac{\partial^{2} V_{\mu}}{\partial \psi^{2}}\right)_{e} .
$$

Since $V_{\mu}$ has a maximum at the equilibrium of interest, the right-hand side of (17) is negative. Therefore $k$ must be chosen to make $A_{\alpha \beta}$ negative at the equilibrium. Define a new control parameter $\tilde{k}$ such that

$$
k=-\left(\frac{1}{3} M+m\right) R^{2}+\frac{\tilde{k}-1}{\tilde{k}}\left(\frac{3}{4} m R^{2}\right) .
$$

Substituting $\tilde{k}$ into (15) and simplifying gives

$$
A_{\alpha \beta}=\frac{1}{3} m l^{2}\left(\frac{\left(\left(\frac{1}{3} m l^{2}-\frac{3}{4} m R^{2}\right) \sin ^{2} \psi-\frac{1}{\tilde{k}} \frac{3}{4} m R^{2}\right)}{\frac{1}{3} m l^{2} \sin ^{2} \psi+\frac{\tilde{k}-1}{\tilde{k}}\left(\frac{3}{4} m R^{2}\right)}\right) .
$$

Then $\left.A_{\alpha \beta}\right|_{e}$ is negative provided $\tilde{k}>1$. When $\tilde{k}>1, A_{\alpha \beta}$ is negative for all $\psi \in(-\bar{\psi}, \bar{\psi})$ where

$$
\bar{\psi}=\sin ^{-1} \sqrt{\left(\frac{1}{\tilde{k}}\right)\left(1+\left(\frac{2 l}{3 R}\right)^{2}\right)^{-1}} .
$$

When $\psi= \pm \bar{\psi}, A_{\alpha \beta}$ becomes zero and the control $u_{\mathrm{cL}}$ becomes singular. Thus $\bar{\psi}$ places a physical limit on the region of attraction of the stabilizing control law. As noted in [3], the value of $\bar{\psi}$ approaches $\frac{\pi}{2}$ in the limit that $\tilde{k} \rightarrow 1$ and $l / R \rightarrow 0$.

For the experimental apparatus, damping which is linear in $\dot{\psi}$ and $\dot{\phi}$ provides a reasonable model of friction. Including the friction model and a dissipative feedback torque $u_{\text {diss }}$, the open-loop equations (14) become

$$
\begin{aligned}
\frac{d}{d t} \frac{\partial L}{\partial \dot{\psi}}-\frac{\partial L}{\partial \psi} & =-d_{\psi} \dot{\psi} \\
\frac{d}{d t} \frac{\partial L}{\partial \dot{\phi}} & =-d_{\phi} \dot{\phi}+u_{c L}+u_{\text {diss }}
\end{aligned}
$$

where $d_{\psi}>0$ and $d_{\phi}>0$. In the notation of Section 3, define

$$
\tilde{d}_{x}^{a b}=\frac{\tilde{d}_{\psi}}{\left(g_{a b}\right)_{e}} \quad \text { and } \quad \tilde{d}_{J}^{a b}=\frac{\tilde{d}_{J}}{\left(g_{a b}\right)_{e}}
$$

where $\tilde{d}_{\psi}$ and $\tilde{d}_{J}$ are dissipative control gains. Let

$$
u_{\mathrm{diss}}=d_{\phi} \dot{\phi}+\left(g_{\alpha b}\right)_{e} \tilde{d}_{\psi} \dot{\psi}-\left(\frac{D_{a b}}{\rho_{a b}}\right)_{e} \tilde{d}_{J} \tilde{J}
$$

with $\tilde{d}_{\psi} \geq 0$ and $\tilde{d}_{J}>0$. Note that the dissipative control law attempts to exactly cancel the damping in the controlled (azimuthal) direction. While exact cancellation is practically impossible, local exponential stability will ensure a degree of robustness to modeling errors.

Figure 2 shows the experimental apparatus. The system is wellmodeled by the equations developed in this section with

$$
\begin{array}{ll}
M=0.259 \mathrm{~kg}, & R=0.211 \mathrm{~m}, \quad d_{\phi}=0.0096 \mathrm{Nms} \\
m=0.130 \mathrm{~kg}, \quad l=0.332 \mathrm{~m}, & d_{\psi}=0.00015 \mathrm{Nms}
\end{array}
$$

One can easily show that choosing the control parameters

$$
\tilde{k}=2, \quad \tilde{d}_{\psi}=10 \mathrm{~s}^{-1}, \quad \tilde{d}_{J}=5 \mathrm{~s}^{-1} .
$$




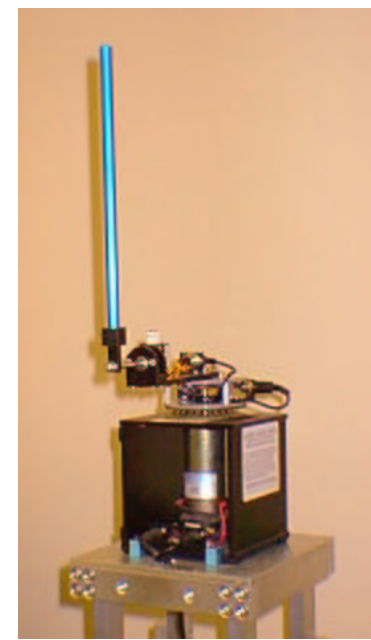

Figure 2: Experimental apparatus.

gives local exponential stability. Figure 3 shows a comparison of experimental and simulated results for these control parameters. The solid curve shows experimental data while the dashed curve shows simulated data with an initial condition determined from the data. At time zero, the pendulum is very near the feedback-stabilized inverted equilibrium. At approximately 2 seconds, the pendulum is perturbed. The system undergoes a damped oscillation, converging once again to near-equilibrium within about 2 seconds.
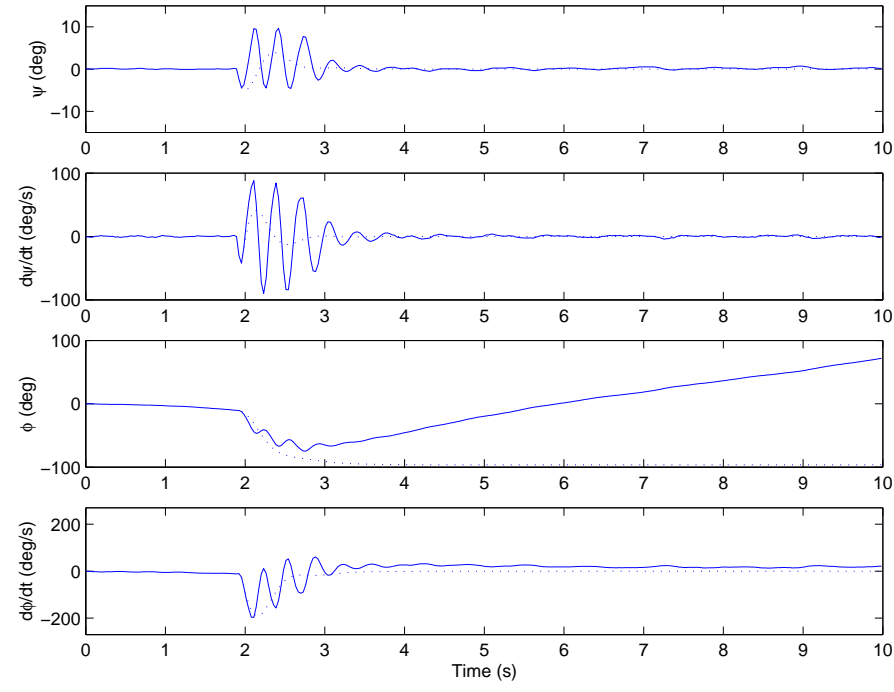

Figure 3: Comparison of experiment and simulation.

One discrepancy between the experiment and the simulation is a slow, steady drift in the $\phi$ direction which is apparent in the latter seconds of the experimental data shown in Figure 3. A likely explanation for the drift is calibration error in the measurement of pendulum elevation $\psi$. Such a bias would naturally induce azimuthal drift. In fact, one should expect drift because the control law preserves the azimuthal symmetry of the system. To eliminate drift, one might employ an additional control torque which breaks this symmetry, as described in [2].

\section{Final Remarks}

The method of controlled Lagrangians provides an algorithmic approach for stabilizing underactuated mechanical systems. For "balance" systems, where the desired equilibrium is a maximum of the potential energy, stabilization involves changing the sign definiteness of the $\tau$-horizontal kinetic energy metric. In such cases, generic physical damping in the unactuated directions enhances stability, while generic damping in the controlled directions is detrimental. Analysis suggests that local exponential stability requires feedback dissipation which "reverses" the effect of damping in the controlled directions.

\section{Acknowledgements}

The authors thank Dong Eui Chang for his helpful comments.

\section{References}

[1] D. Auckley, L. Kapitanski, W. White. "Control of nonlinear underactuated systems", Comm. Pure and Applied Mathematics, 53, pp. 354-369, (2000).

[2] A. M. Bloch, D. E. Chang, N. E. Leonard, J. E. Marsden. "Controlled Lagrangians and the stabilization of mechanical systems II: Potential shaping and tracking", IEEE Transactions on Automatic Control (To appear.)

[3] A. M. Bloch, N. E. Leonard, J. E. Marsden. "Stabilization of the pendulum on a rotor arm by the method of controlled Lagrangians", Proc. Int. Conf. on Robotics and Automation, pp. 500-505, IEEE (1999).

[4] A. M. Bloch, N. E. Leonard, J. E. Marsden. "Controlled Lagrangians and the stabilization of mechanical Systems I: The first matching theorem", IEEE Transactions on Automatic Control, 45:12, pp. 2253-2270, (2000).

[5] J. Hamberg. "General matching conditions in the theory of controlled Lagrangians", Proc. IEEE Conf. Decision and Control, 38 pp. 2519-2523, Phoenix, AZ (December 1999).

[6] R. Ortega, A. van der Schaft, B. Maschke, G. Escobar. "Energy-shaping of port-controlled Hamiltonian systems by interconnection", Proc. IEEE Conf. Decision and Control, 38 pp. 1646-1651, Phoenix, AZ (December 1999).

[7] A. J. van der Schaft "Stabilization of Hamiltonian systems", Nonlinear Analysis: Theory, Methods, and Applications, 10:10 pp. 1021-1035, (1986).

[8] C. Woolsey, N. E. Leonard. "Global asymptotic stabilization of an underwater vehicle using internal rotors", Proc. IEEE Conf. Decision and Control, 38 pp. 25272532, Phoenix, AZ (December 1999). 